\title{
Tamaño, color de nuez y sombra afectan la
}

\section{germinación de Quercus deserticola}

Nut size and color and shade affect germination of Quercus deserticola

\author{
Dante Arturo Rodríguez-Trejo y yarín Pompa-García²
}

1 Laboratorio de Semillas Forestales, División de Ciencias Forestales, Universidad Autónoma Chapingo, Chapingo, Edo. de México.

\author{
2 Facultad de Ciencias Forestales, Universidad Juárez * Autor de correspondencia. dantearturo@yahoo.com \\ del Estado de Durango. Durango, México. \\ mpgarcia@ujed.mx
}

\section{RESUMEN}

México es el país más rico en especies del género Quercus del planeta, con 157 de ellas. Los encinos tienen una gran importancia ecológica y potencial económico. No obstante, sus semillas y propagación han sido poco estudiadas. El objetivo del presente trabajo fue estudiar la influencia de tamaño y color de la nuez, así como de la sombra, en la capacidad y energía germinativas de Quercus deserticola. Para ello se instaló un experimento en cámara de ambiente controlado $\left(25^{\circ} \mathrm{C} / 20{ }^{\circ} \mathrm{C}\right)$, con un fotoperiodo de $10 \mathrm{~h}$ luz. Los factores fueron tamaño de la nuez (chica y grande), color (café claro y café oscuro) y sombra ( $40 \%$ ) (con y sin). El diseño experimental fue en bloques al azar, anidados dentro del factor sombra. Se condujo un análisis de varianza con un modelo mixto y una comparación de medias de Tukey, para las variables capacidad germinativa y energía germinativa, y se obtuvieron los parámetros de un modelo logístico para estimar la probabilidad de germinación. Se halló un efecto significativo de tamaño ( $\mathrm{p}=0.0067$ ) y color $(\mathrm{p}<0.0001)$ de la semilla, así como de la sombra $(\mathrm{p}=0.0078$ ) en la capacidad germinativa. La mayor capacidad germinativa, de 100\%, se logró con semilla de nuez grande, café oscuro y con sombra. De acuerdo con el análisis de varianza, la energía germinativa (número de días para alcanzar $70 \%$ de la capacidad germinativa) fue influida por el color $(\mathrm{p}<0.0001)$ y por la interacción tamaño* color $(\mathrm{p}=0.0351)$. La mayor energía germinativa, con un valor de 11.5 días, se alcanzó con semilla de nuez grande y café oscura.

Palabras Clave: análisis de semillas, encinos, semillas forestales.

\section{ABSTRACT}

Mexico is the country with the highest number of Quercus species worldwide (157). The oaks have great ecological importance and economic potential. However, their seeds and nursery propagation have been scarcely studied. The aim of this study was to investigate the effect of nut size and color, as well as shade, on germination of Quercus deserticola. The experiment was installed in a controlled environment chamber $\left(25^{\circ} \mathrm{C} / 20^{\circ} \mathrm{C}\right.$, with a photoperiod of $10 \mathrm{~h}$ of light). The experimental factors were nut size (small and large), color (light brown and dark brown), and shade (with and without $40 \%$ shade). The experimental design was completely randomized blocks, with the blocks nested into the shade factor. An analysis of variance with a mixed procedure and a Tukey means comparison were conducted, with the variables germinative capacity and germinative energy and a logistic model was constructed to estimate the probability of germination. There was a significant effect of seed size $(\mathrm{p}=0.0067)$, seed color $(\mathrm{p}<0.0001)$ and shade $(\mathrm{p}=0.0078)$ on germinative capacity. The highest germinative capacity $(100 \%)$ was for large seeds of dark brown color and under shade. The germinative energy (number of days to reach $70 \%$ of the germinative capacity) was affected by seed color $(\mathrm{p}<0.0001)$ and by the interaction seed size ${ }^{*}$ color $(\mathrm{p}=0.0267)$. The highest germinative energy (11.5 days) was for large and dark brown colored seed.

KEYWORDS: seed testing, oaks, forest seeds.

\section{INTRODUCCIÓN}

De todo el planeta, México es el país con la mayor riqueza de especies del género Quercus, con 157 de ellas (Zavala, 2001). Se trata de un género con gran relevancia ecológica, debido a su amplia extensión en las zonas montañosas del país, y también a que con frecuencia precede tanto altitudinalmente como en la sucesión ecológica al género Pinus, con el que además forma frecuentes asociaciones (Rzedowski, 1978).

Quercus deserticola Trel. es una especie endémica de México, que se halla en doce estados, entre 2000 m y 3000 $\mathrm{m} \mathrm{snm}$, conformando principalmente bosques de encino y 
asociándose con matorrales. Es un árbol de $2.5 \mathrm{~m}$ a $10 \mathrm{~m}$ de altura y diámetro normal de $12 \mathrm{~cm}$ a $70 \mathrm{~cm}$; su corteza es café oscura, con surcos muy marcados; hojas oblongooblanceoladas a lanceoladas u ovaladas, con $3 \mathrm{~cm}$ a $16 \mathrm{~cm}$ de longitud $1.2 \mathrm{~cm}$ a $5.0 \mathrm{~cm}$ de anchura, el haz verde oscuro, algo brillante y rugoso, el envés claro con abundantes tomentos (Zavala, 2003; Arizaga, Martínez-Cruz, SalcedoCabrales y Bello-Gonzáles, 2009). Esta especie es empleada para obtención de leña y carbón, fabricación de postes, horcones, cabos, arados, así como en el curtido de pieles y para la obtención de celulosa para papel (Arizaga et al., 2009).

Existe variabilidad en el tamaño de la semilla de cualquier especie, si bien en algunas esto es más marcado que en otras. Las semillas grandes de diversos géneros, tienden a germinar más pronto y a desarrollar plántulas de mayor tamaño. También el color de la semilla puede variar en una misma especie, representando ventajas ante diferentes condiciones ambientales (Baskin y Baskin, 2001). Por ejemplo, las semillas de Cecropia obtusifolia Bertol. de color café claro tienen mayor capacidad germinativa a mayor temperatura que las semillas color café oscuro y estas últimas germinan mejor que las primeras a bajas temperaturas (Tenorio-Galindo, Rodríguez-Trejo y López-Ríos, 2008).

Las especies pioneras tienden a ser intolerantes a la sombra, mientras que las de etapas sucesionales más avanzadas normalmente son tolerantes a dicho factor. En el caso de la especie bajo estudio, se considera que es inicial o intermedia (Zavala, 2007), por lo cual podría ser intolerante o indiferente a la presencia o ausencia de sombra.

La vulnerabilidad de los ecosistemas ante las variaciones climáticas y efectos antropogénicos propician una oportunidad para mejorar el entendimiento de los mecanismos ecológicos que enfrentan las especies (Dawson, Jackson., House, Prentice y Mace 2011). Este conocimiento puede resultar fundamental en potenciar el uso y propagación de Q. deserticola. Esta especie ha recibido poca atención por parte de los investigadores, a pesar del gran potencial que tiene para ser utilizada en programas de reforestación y restauración ecológica de bosques de encino y matorrales donde también se le encuentra.

\section{OBJETIVOS E HIPÓTESIS}

El objetivo del presente trabajo fue realizar el análisis de la semilla de Q. deserticola y probar el efecto del tamaño y color de la nuez, así como el de la sombra en la capacidad y energía germinativa de la semilla. La hipótesis es que la semilla grande, color café oscuro y bajo sombra, tendrá mejor: capacidad germinativa, probabilidad de germinación y energía germinativa.

\section{MATERIALES Y MÉTODOS}

En octubre de 2015 fueron recolectados $4 \mathrm{~kg}$ de nueces maduras de Q. deserticola de diez árboles con $20 \mathrm{~cm}$ a 40 $\mathrm{cm}$ de diámetro normal y $6 \mathrm{~m}$ a $10 \mathrm{~m}$ de altura, sanos y $\sin$ daños evidentes, cerca del poblado de San Nicolás Tlaminca, Municipio de Texcoco, Estado de México. Las coordenadas de la zona de recolección son: $19^{\circ} 29^{\prime} 47.97$ ' $\mathrm{N}$, 98 48'51.98”O; $19^{\circ} 29^{\prime} 48.81^{\prime \prime} \mathrm{N}, \quad 98^{\circ} 48^{\prime} \quad$ 57.87”O; $19^{\circ} 29^{\prime} 49.12^{\prime \prime N}, \quad 98^{\circ} 48^{\prime} 52.60^{\prime \prime O}$ y $19^{\circ} 29^{\prime} 50.11^{\prime \prime} \mathrm{N}$, 98 48'57.85"O. Aproximadamente la mitad del germoplasma fue obtenido de las copas de los árboles y la mayoría tenía un color café oscuro. La otra mitad fue recogida del suelo evitando la que ya mostraba signos de deterioro, pudrición o perforaciones por salida de larvas de coleópteros, el color de la mayor parte era café claro. De las nueces recolectadas, $1.5 \mathrm{~kg}$ se utilizaron como muestra de trabajo para el presente experimento.

Para la estimación del peso, se contaron y pesaron 100 nueces color café oscuro, pues tal color es el del fruto que recientemente ha madurado. El peso fue convertido a número de unidades por kilogramo y como el peso de 1000 semillas. Con el propósito de obtener el contenido de humedad, una submuestra de $72.9 \mathrm{~g}$ (34 nueces) fue puesta en horno de secado a $75{ }^{\circ} \mathrm{C}$, hasta obtener el peso anhidro (peso constante). Esta variable se obtuvo tanto con base en peso anhidro $\left(\mathrm{CH}_{\mathrm{o}}\right)$ como con base en peso verde $\left(\mathrm{CH}_{\mathrm{v}}\right)$, de acuerdo con los modelos 1 y 2 .

$\mathrm{CH}_{\mathrm{o}}=\left(P_{v}-P_{\mathrm{o}}\right) / P_{\mathrm{o}}$ 
$\mathrm{CH}_{v}=\left(P_{v}-P_{\mathrm{o}}\right) / P_{v}$

Donde: $P_{v}$ es el peso en verde y $P_{\mathrm{o}}$ el peso anhidro $(\mathrm{g})$

Fue instalado un experimento en cámaras de ambiente controlado, con un régimen día/noche de $25{ }^{\circ} \mathrm{C} / 20{ }^{\circ} \mathrm{C}$ y un fotoperiodo de $10 \mathrm{~h}$, con luz fluorescente (radiación fotosintéticamente activa media $\left.=134.7 \mu \mathrm{mol} \mathrm{m}^{2} \mathrm{~s}^{-1}\right)$. Las siembras se hicieron en cajas de plástico de $3.5 \mathrm{~L}$ con tapa y como sustrato se utilizó tela de paño. El paño quedó sobre una retícula de plástico con soportes y un extremo de la tela se dejó en contacto con el fondo de la caja y el agua, por lo cual no fue necesario regar más que inicialmente las semillas, con $250 \mathrm{~mL}$ de una solución de agua destilada con el fungicida Captán $\left(2 \mathrm{~g} \mathrm{~L}^{-1}\right)$.

La nuez fue clasificada en chica $(\leq 1.70 \mathrm{~cm}$ de longitud de bellota) y grande $(>1.70 \mathrm{~cm})$, así como por su color en café oscuro y café claro. Parte de las nueces fueron sembradas bajo una sombra de $40 \%$, la cual se aplicó forrando las cajas correspondientes con tela de tul verde a doble capa. La semilla fue considerada germinada cuando la radícula igualó la longitud de la nuez. Las semillas sembradas en la condición sombreada siempre fueron mantenidas en tal condición, incluso el registro de su germinación se hizo en cuarto con luz tenue. El experimento duró 41 días: fue instalado el 30 de octubre y finalizado el 10 de diciembre de 2015. La germinación fue evaluada diariamente. También se evaluó la energía germinativa, como el número de días para alcanzar $70 \%$ de la capacidad germinativa.

El diseño experimental fue en bloques completamente al azar, con seis repeticiones y con los bloques anidados dentro del factor sombra, de acuerdo con el modelo 3. Cada repetición constó de 12 nueces y cada bloque de 48 de ellas.

$\mathrm{y}_{\mathrm{ijk} \mathrm{k}}=\mu+\alpha_{\mathrm{i}}+\beta_{\mathrm{j}}+\gamma_{\mathrm{k}}+\gamma_{\mathrm{k}}(\zeta)_{1}+(\alpha \beta)_{\mathrm{ij}}+(\alpha \gamma)_{\mathrm{ik}}+\alpha_{\mathrm{i}} \gamma_{\mathrm{k}}(\zeta)_{1}+(\beta \gamma)$

${ }_{\mathrm{jk}}+\beta_{\mathrm{i}} \gamma_{\mathrm{k}}(\zeta)_{1}+(\alpha \beta \gamma)_{\mathrm{ijk}}+\alpha_{\mathrm{i}} \beta_{\mathrm{j}} \gamma_{\mathrm{k}}(\zeta)_{1}+\varepsilon_{\mathrm{ijk} \mathrm{l}}$

Donde: $\mu=$ media general, $\alpha_{\mathrm{i}}=$ efecto del i-ésimo nivel del factor tamaño de la nuez, $\beta_{\mathrm{j}}=$ efecto del $\mathrm{j}$-ésimo nivel del factor color de la nuez, $\gamma_{k}=$ efecto del k-ésimo nivel del factor sombra, $\gamma_{k}(\zeta)_{1}=$ efecto del l-ésimo bloque, anidado dentro del k-ésimo nivel del factor sombra, $(\alpha \beta)_{\mathrm{ij}}=$ interacción entre el i-ésimo nivel del factor tamaño de nuez con el j-ésimo nivel del factor color de nuez, $(\alpha \gamma)_{\mathrm{ik}}=$ interacción entre el i-ésimo nivel del factor tamaño de nuez y el k-ésimo nivel del factor sombra, $\alpha_{\mathrm{i}} \gamma_{\mathrm{k}}(\zeta)_{1}=$ interacción entre el i-ésimo nivel del factor tamaño de nuez y el l-ésimo bloque anidado dentro del k-ésimo nivel del factor sombra, $(\beta \gamma)_{\mathrm{jk}}=$ interacción entre el j-ésimo nivel del factor color de la nuez y el k-ésimo nivel del factor sombra, $\beta_{\mathrm{j}} \gamma_{\mathrm{k}}(\zeta)$ ${ }_{1}=$ interacción entre $\mathrm{j}$-ésimo nivel del factor tamaño de nuez y el l-ésimo bloque anidado dentro del k-ésimo nivel del factor sombra, $(\alpha \beta \gamma)_{\mathrm{ijk}}=$ efecto de la interacción entre el i-ésimo nivel del factor tamaño de la nuez con el j-ésimo nivel del factor color de la nuez y el k-ésimo nivel de sombra, $\alpha_{\mathrm{i}} \beta_{\mathrm{j}} \gamma_{\mathrm{k}}(\zeta)_{1}=$ efecto de la interacción entre el i-ésimo nivel del factor tamaño de la nuez, el j-ésimo nivel del factor color de la nuez y el l-ésimo bloque, anidado dentro del $\mathrm{k}$-ésimo nivel del factor sombra, $\varepsilon_{\mathrm{ijkl}}=$ error experimental $\left(0, \sigma^{2}\right)$. Los efectos de los factores tamaño y color de nuez, así como sombra, son fijos, los bloques y todas sus interacciones y anidaciones con efectos aleatorios.

Con el propósito de obtener la probabilidad de germinación de las semillas (P), se utilizó un modelo logístico (4).

$\mathrm{P}=1 /\left(1+\mathrm{e}-\left({ }^{\alpha+\beta x_{1}+\beta x_{2}+\beta x_{3}}\right)\right)$

Donde: $\mathrm{e}=$ base de los logaritmos naturales, $\alpha=$ ordenada al origen, $\beta=$ parámetro asociado a la variable independiente $\mathrm{X}_{1}$ (tamaño de la nuez, grande $=1$, chica $\left.=0\right), \beta_{2}=$ constante asociada a la variable independiente $\mathrm{X}_{2}$ (color de la nuez, café oscuro $=1$, café claro $=0), y \beta_{3}=$ constante asociada a la variable independiente $\mathrm{X}_{3}$ (sombra, con sombra $=1$, sin sombra $=0$ ).

Para el análisis estadístico se utilizó el procedimiento mixto (Proc Mixed) de SAS para microcomputadoras, v. 9.0. Los grados de libertad se calcularon con el método Satterthwaite. También se llevó a cabo una prueba de 
comparación de medias de Tukey, utilizando el procedimiento ANOVA de SAS. En los análisis anteriores, por ser binomial la variable germinación fue transformada con la función (5):

$$
G_{t}=\operatorname{arcsen}(G)^{0.5}
$$

Donde: $G_{t}=$ germinación transformada, arcsen $=$ arco seno, $G=$ germinación (en tanto por uno). Para facilitar la comprensión de los resultados de la comparación de medias, se utilizaron los valores de germinación final sin transformar.

En el caso del análisis de probabilidad de germinación, fue empleado el procedimiento logistic (Proc Logistic), también de SAS. A este modelo se le aplicó la prueba de bondad de ajuste de Hosmer-Lemeshow. Además se verificó que el intervalo de confianza no contuviera el valor 1 y que la concordancia fuera alta, mayor a $60 \%$. Para el análisis mixto, la prueba de comparación de medias de Tukey y la significancia del modelo mixto y sus variables, fue considerada $\mathrm{p} \leq 0.05$.

\section{Resultados}

\section{Descripción de la semilla}

Las nueces mostraron una importante variación en tamaño, entre $1.36 \mathrm{~cm}$ y $2.30 \mathrm{~cm}$ de longitud, aunque Vázquez (1992) refiere nueces más pequeñas para esta especie, de hasta $0.8 \mathrm{~cm}$. Las partes principales de nuez y semilla se muestran en la figura 1.

\section{Pruebas físicas}

Como en el laboratorio se trabajó con nueces limpias, la pureza fue de $100 \%$. El peso de mil nueces fue igual a $2305.6 \mathrm{~g}$; se tuvieron 434 nueces $\mathrm{kg}^{-1}$. El contenido de humedad, base anhidra, fue de $105 \%$, en tanto que con base en verde alcanzó $51.2 \%$, lo que confirma que se trata de una semilla recalcitrante.

\section{Germinación}

Los factores tamaño de nuez $(\mathrm{p}=0.0067)$, color de nuez $(<0.0001)$ y sombra $(\mathrm{p}=0.0078)$ resultaron estadísticamente significativos para la capacidad germinativa. La prueba de comparación de medias mostró que las semillas grandes, color café oscuro y con sombra, germinaron mejor que sus contrapartes pequeñas, color café claro y
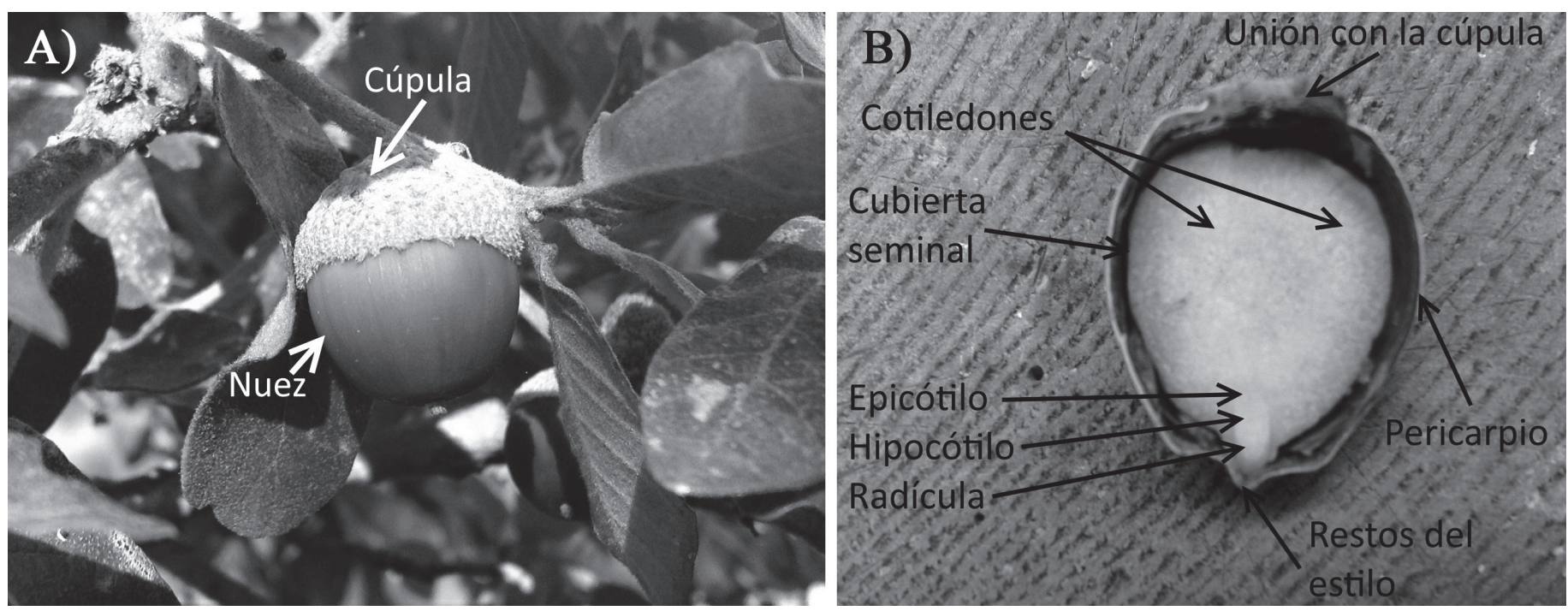

Figura 1. Principales partes de fruto y semilla de Quercus deserticola. A) Vista exterior de la nuez. B) Corte longitudinal de fruto y semilla. 
sin sombra (Tabla 1). Las curvas de germinación acumulada se muestran en la figura 2 .

También se hizo un análisis considerando las combinaciones de los diferentes niveles de los factores como tratamientos independientes y resultó significativo $(\mathrm{p}<$ 0.0001). El tratamiento nueces grandes, color café oscuro y con sombra, alcanzó $100 \%$ de capacidad germinativa;

TABla 1. Resultados de la prueba de comparación de medias de Tukey, para capacidad germinativa, en relación con los tres factores bajo estudio.

\begin{tabular}{ccc}
\hline Factor & Nivel & Capacidad germinativa (\%) \\
\hline \multirow{2}{*}{ Tamaño de nuez } & Grande & $77.5 \mathrm{a}$ \\
& Pequeña & $70.6 \mathrm{~b}$ \\
\hline \multirow{2}{*}{ Color de nuez } & Café oscuro & $92.9 \mathrm{a}$ \\
& Café claro & $55.2 \mathrm{~b}$ \\
\hline \multirow{2}{*}{ Sombra } & Con & $77.1 \mathrm{a}$ \\
& Sin & $71.0 \mathrm{~b}$ \\
\hline
\end{tabular}

Pares de niveles de factores con letra distinta tuvieron diferencias significativas $(p<0.05)$ entre sí.

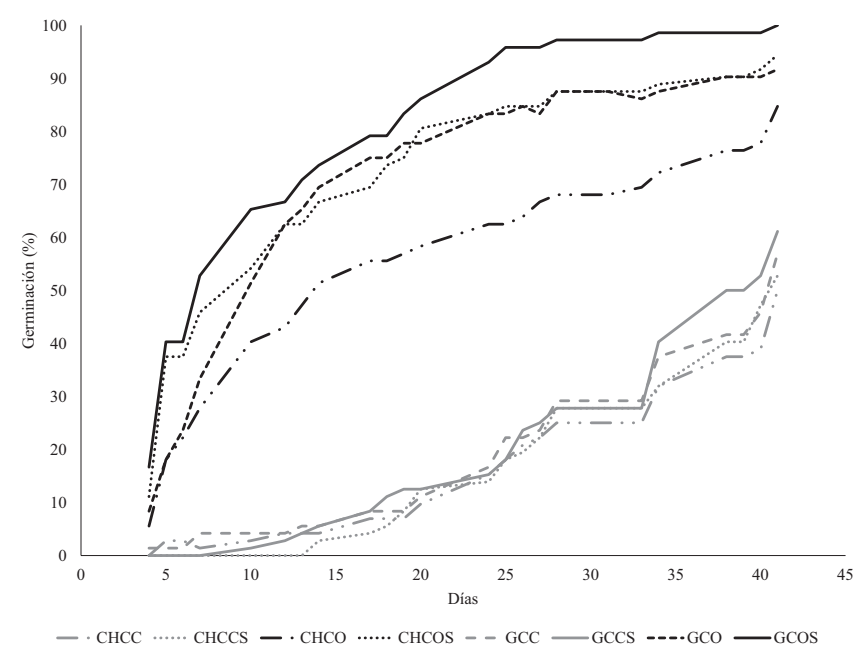

Figura 2. Curvas de germinación acumulada en Quercus deserticola, para los diferentes tratamientos. $\mathrm{CH}=$ nuez chica, $\mathrm{G}=$ nuez grande, $\mathrm{CC}=$ color café claro, $\mathrm{CO}=$ color café oscuro, $\mathrm{S}=$ con sombra. en tanto que el tratamiento con semillas pequeñas, color café claro y sin sombra, solo tuvo $50 \%$ (Tabla 2).

\section{Energía germinativa}

La energía germinativa tuvo diferencias significativas en los factores color $(\mathrm{p}<0.0001)$ y para la interacción tamaño*color ( $\mathrm{p}=0.0267)$. La prueba de comparación de medias de Tukey arrojó diferencias entre colores $(\mathrm{p}<$ 0.05), con promedios iguales a 14.5 días para la nuez café oscuro y 34.2 días para la nuez color café claro. Se halló la mayor energía germinativa en las nueces grandes y color café oscuro, con 11.5 días para alcanzar $70 \%$ de la capacidad germinativa. La interacción tamaño*color se muestra en la figura 3.

\section{Probabilidad de germinación}

En relación con la probabilidad de germinación, los factores individuales fueron significativos. No así la totalidad de las interacciones $(\mathrm{p}>0.05)$, por lo cual fueron eliminadas del modelo, volviendo a realizar al análisis, cuyos resultados se muestran a continuación. La significancia de las variables se muestra en la tabla 3. La prueba de bondad de ajuste de Hosmer-Lemeshow arrojó una $\mathrm{p}=$ 0.3309 , lo cual significa que el modelo está correctamente especificado.

TABla 2. Comparación de medias para capacidad germinativa de Q. deserticola, considerando combinaciones de niveles de factores como tratamientos.

\begin{tabular}{cc}
\hline Tratamiento & Capacidad germinativa (\%) \\
\hline GCOS & $100.0 \mathrm{a}$ \\
CHCOS & $94.7 \mathrm{ab}$ \\
GCON & $91.8 \mathrm{ab}$ \\
CHCON & $85.0 \mathrm{~b}$ \\
GCCS & $61.0 \mathrm{c}$ \\
GCCN & $57.0 \mathrm{c}$ \\
CHCCS & $52.8 \mathrm{c}$ \\
CHCCN & $50.0 \mathrm{c}$ \\
\hline
\end{tabular}

$\mathrm{G}=$ nuez grande, $\mathrm{CH}=$ nuez chica, $\mathrm{CO}=$ café oscuro, $\mathrm{CC}=$ café claro, $\mathrm{S}=$ con sombra, $\mathrm{N}=\sin$ sombra. 


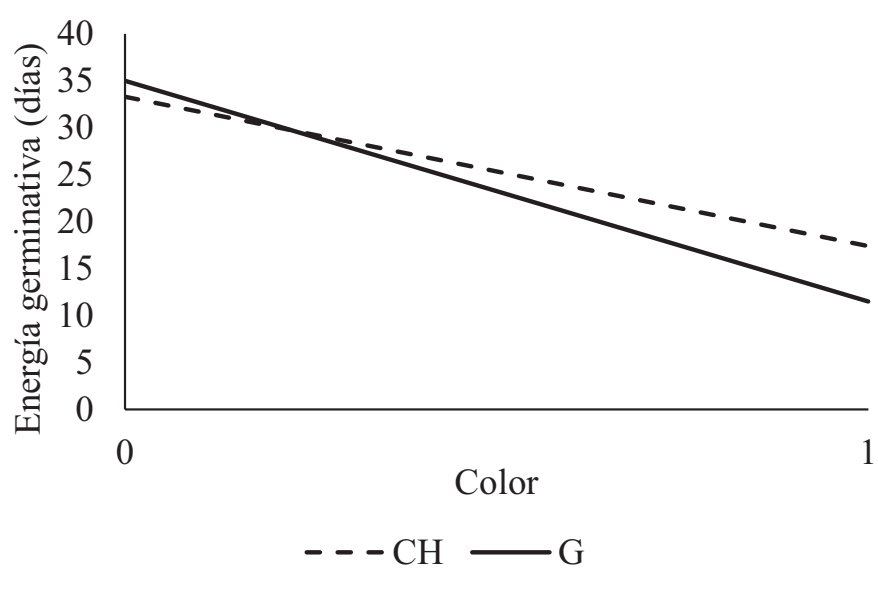

Figura 3. Interacción tamaño y color de la nuez para la energía germinativa de la semilla de Q. deserticola. Tamaño: $\mathrm{CH}=$ chica, $\mathrm{G}$ = grande. Color: 0 = café claro, 1 = café oscuro.

El modelo obtenido fue (6):

$\mathrm{P}=1 /\left(1+\mathrm{e}^{-\left(1.3719+0.2326 \mathrm{X}_{1}+1.1582 \mathrm{X}_{2}+0.1886 \mathrm{X}_{3}\right)}\right)$

De esta forma, la probabilidad de germinación para semillas grandes, café oscuras, con el nivel de sombra y condiciones planteadas para este experimento, alcanza $55.2 \%$; en cambio para semillas pequeñas, color café oscuro y sin sombra, tal valor es $20.2 \%$ (Tabla 4 ).

\section{DISCUSIÓN}

El contenido de humedad letal de la semilla en muchos Quercus de Estados Unidos va de $15 \%$ a $20 \%$ en encinos rojos y negros, y de $25 \%$ a $30 \%$ en los blancos (Bonner, 2008). La semilla del encino estudiado, que es del subgénero de los blancos, tuvo un contenido de humedad mayor (51.2\%), cifra dentro del intervalo de $40 \%$ a $50 \%$, considerado como seguro por Bonner (2008) y con potencial para almacenar a $1{ }^{\circ} \mathrm{C}-3{ }^{\circ} \mathrm{C}$ semillas de encinos blancos, por 0.5 a 3 años. Zavala (2004) señala que aunque el contenido de humedad de la semilla de Q. deserticola sea reducido a poco menos de $50 \%$, germinará bien. Además, puntualiza que se puede almacenar en temperaturas de 3
TABla 3. Significancia de intercepto y variables para el modelo logístico.

\begin{tabular}{|c|c|c|}
\hline Variable & $\mathrm{p}$ & $\begin{array}{c}\text { Intervalo de confianza } \\
\text { al } 95 \%\end{array}$ \\
\hline Intercepto & $<0.0001$ & \\
\hline Tamaño & 0.0281 & 1.05 a 2.413 \\
\hline Color & $<0.0001$ & 6.615 a 16.675 \\
\hline Sombra & 0.0746 & 0.963 a 2.208 \\
\hline
\end{tabular}

Tabla 4. Probabilidad de germinación para semillas de $Q$. deserticola, en función del tamaño de semilla, su color y sombra.

\begin{tabular}{cccc}
\hline Tamaño & Color & Sombra & P (\%) \\
\hline Grande & Café oscuro & Con & 55.2 \\
Grande & Café oscuro & Sin & 50.5 \\
Grande & Café claro & Con & 27.9 \\
Grande & Café claro & Sin & 24.2 \\
Pequeña & Café oscuro & Con & 49.4 \\
Pequeña & Café oscuro & Sin & 44.6 \\
Pequeña & Café claro & Con & 23.4 \\
Pequeña & Café claro & Sin & 20.2 \\
\hline
\end{tabular}

${ }^{\circ} \mathrm{C}$ a $7{ }^{\circ} \mathrm{C}$ por más de seis meses, luego de los cuales su viabilidad será de por lo menos $50 \%$.

La variación en tamaño de la semilla, dada por la relación de tamaño de la mayor semilla hallada entre el tamaño de la más pequeña encontrada, fue igual a 1.69, de modo que según Leishman, Wright, Moles y Westoby (2000) tal relación es menor a un orden de magnitud y variación intraespecífica típica. No obstante, Vázquez (1992) refiere semillas tan pequeñas como $0.8 \mathrm{~cm}$ de longitud, con lo cual a nivel de especie la relación mencionada alcanza 2.87, cifra que rebasa un orden de magnitud (valor $=2$ ).

Dicha variabilidad de tamaño es común en el género, tanto entre especies como entre poblaciones y dentro de un mismo árbol. Por ejemplo, Márquez, Mendizábal-Hernández y Flores (2005) hallaron variabilidad significativa 
en largo, ancho y peso de las semillas de Q. oleoides Schltdl. \& Cham. del centro de Veracruz, tanto entre diferentes sitios como entre árboles.

La mayor germinación de semillas grandes de $Q$. deserticola corresponde con lo que Bonner (2008) documenta para varias semillas del género en Estados Unidos y con los resultados de Huerta y Rodríguez (2011) para Quercus rugosa de México central. Esto se debe a que las plántulas de semillas grandes son más capaces de emerger desde mayores profundidades que las de semillas pequeñas (Baskin y Baskin, 2001). Además, las semillas grandes de encinos producen un sistema radical más extenso y profundo que los de semillas más pequeñas, lo cual les permite obtener agua más eficientemente en ambientes con limitaciones de humedad (Zavala y García, 1996). De forma similar al presente trabajo, las semillas pesadas de Quercus dealbata L. y Q. griffithii Hk. f. \& Th., de un bosque tropical seco de la India, germinan más rápido que las ligeras, si bien no necesariamente las semillas más pesadas eran las más grandes (Tripathi y Khan, 1990).

Respecto a que las semillas de nueces color café oscuro germinaron mejor que las de color café claro, la variación en color hallada en la especie estudiada se relaciona con el nivel de madurez. Al madurar, las nueces pasan del color verde al café oscuro y después lo van perdiendo, se van haciendo más pálidas. La especie estudiada comienza la producción de bellotas en octubre y la recolección se hizo a fines de ese mes, por lo cual se estima que la edad de las nueces color café claro que estaban en el piso forestal, no excedía de tres a cuatro semanas. El nivel de palidez puede estar relacionado con la pérdida de viabilidad en estas semillas recalcitrantes, la cual es del orden de seis meses.

Debido a que la semilla de Q. deserticola bajo sombra germinó más que la expuesta, se evidencia una ligera fotoinhibición. De acuerdo con Pons (2000), en algunas especies cuya semilla yace en la superficie del suelo se evita la germinación en sitios expuestos, donde las condiciones no son adecuadas para el establecimiento, en particular por limitaciones de humedad, aunque esta relación entre luz y humedad no está esclarecida del todo. De la misma forma, Zavala (2001) señala que los encinos crecen en zonas con sequías estivales y las plántulas se establecen a la sombra de ellos o de plantas del sotobosque, por lo cual las plántulas de encinos compiten con las plantas que lo componen. El producir pocas semillas grandes les ha conferido ventaja adaptativa sobre las especies que producen semilla pequeña. Por otra parte, las especies con semilla pequeña tienen mayor mortalidad en condiciones de sombra (Lesishman et al., 2000).

Q. deserticola se halla normalmente en terrenos rocosos de colinas secas o semisecas, rocosas. La especie es común en bosques de encino y de oyamel, en áreas abiertas o perturbadas (Zavala, 2007). En esas localidades, la sombra de la misma copa del árbol madre puede generar un microclima favorable, que prevenga la deshidratación, favoreciendo la germinación de la semilla.

Hay evidencia de que la germinación de especies de Quercus con semilla grande se ve favorecida por la sombra, mientras que en las especies con semilla pequeña favorece la exposición a la radiación solar directa (Zavala y García, 1996). Esta tendencia se halló en San Luis Potosí, al comparar Q. coccolobifolia Trel., que tiene semilla pequeña $(0.8 \mathrm{~cm}$ a $1.0 \mathrm{~cm})$ y que se regenera más a plena luz con Q. affinis Scheidw., el cual tiene semilla más grande $(0.9 \mathrm{~cm}$ a $1.3 \mathrm{~cm})$ y cuya regeneración se da mejor bajo sombra (Castillo, Flores, Aguirre y Yeaton, 2008). Sin embargo, a nivel intraespecífico en la población aquí estudiada de Q. deserticola no se observó esa tendencia para la capacidad germinativa.

A una procedencia de Q. deserticola de la Mixteca Alta, Oaxaca, se le registró germinación de $53 \%$ a $18{ }^{\circ} \mathrm{C}$ y con un fotoperiodo de 12 h (Martínez-Pérez, OrozcoSegovia y Martorell, 2006). Comparativamente los resultados del presente trabajo fueron superiores bajo las condiciones del mejor tratamiento: $25^{\circ} \mathrm{C} / 20^{\circ} \mathrm{C}$, fotoperiodo de $10 \mathrm{~h}$ y semillas grandes, color café oscuro y bajo sombra. Incluso los tratamientos con menor germinación prácticamente igualaron los de la referencia señalada.

La interacción entre tamaño de la semilla y su color, incide en la energía germinativa de la especie bajo estudio y, de acuerdo con Huerta y Rodríguez (2011), el pri- 
mer factor, en interacción con la temperatura, influye en la de Q. rugosa. En ambos estudios la semilla grande tuvo mayor energía germinativa, es decir, requirió de menos tiempo para alcanzar $70 \%$ de la capacidad germinativa. De manera similar a la capacidad germinativa, después de la maduración, conforme la nuez pierde intensidad en su color, la energía germinativa también se reduce, es decir, requiere más días para alcanzar $70 \%$ de la capacidad germinativa y esto es más evidente en las semillas pequeñas.

\section{CONCLUSIONES}

Como en otras especies de encinos, las semillas de mayor tamaño de Q. deserticola exhibieron una capacidad germinativa superior. Las nueces color café claro son una etapa de madurez posterior a las de color café oscuro, y tuvieron semillas con una menor germinación. Por ello, es mejor utilizar nueces oscuras en los viveros forestales. Si bien este encino es considerado de etapas sucesionales iniciales, mostró una ligera fotoinhibición a la germinación, ya que una sombra ligera (de $40 \%$ ) promovió la germinación. Las semillas grandes, de nueces oscuras y bajo sombra ligera alcanzaron la máxima capacidad germinativa $(100 \%)$, mientras que las semillas pequeñas, de nueces color café claro y sin sombra, solamente alcanzaron la mitad de dicha germinación. También con las nueces grandes, color café oscuro y bajo sombra ligera se tuvo la mayor probabilidad de germinación, en tanto que la mejor energía germinativa se logró con nueces grandes, color café oscuro.

\section{REFERENCIAS}

Arizaga, S., Martínez-Cruz., J., Salcedo-Cabrales, M. y BelloGonzáles, M. A. (2009). Manual de la biodiversidad de encinos michoacanos. México: Semarnat, INE.

Baskin, C. C. y Baskin, J. M. (2001). Seeds. Ecology, biogeography, and evolution of dormancy and germination. EUA: Academic Press.

Bonner, F. T. (2008). Quercus. L. Oak. En F.T. Bonner y R. P. Karrfalt (Eds.), The Woody Plant Seed Manual. Agricul- ture Handbook 727(pp. 928-938). Washington D.C.: USDA Forest Service.

Castillo, L. P., Flores C., J. A., Aguirre R., J. R. y Yeaton H., R. I. (2008). Dinámica sucesional del encinar de sierra de Álvarez, S. L. P. Madera y Bosques, 14 (1), 21-35.

Dawson, T. P., Jackson., S. T., House., J. I., Prentice, I. C. y Mace, G. M. (2011). Beyond predictions: biodiversity conservation in a changing climate. Science, 332, 53-58.

Huerta P., R. y Rodríguez-Trejo, D. A. (2011). Tamaño de semilla y temperatura: sus efectos en la germinación de Quercus rugosa Née. Revista Chapingo. Serie Ciencias Forestales y del Ambiente, 17 (2), 179-187.

Leishman, R. L., Wright., I. J., Moles, A. T. y Westoby, M. (2000). The evolutionary ecology of seed size. En M. D. Fenner (Ed.) Seeds. The ecology of regeneration in plant communities (2nd ed.) (pp. 31-57). United Kingdom: Cabi Pub.

Márquez, R. J., Mendizábal-Hernández, L. C. y Flores R., C. I. (2005). Variación en semillas de Quercus oleoides Schl. et Cham. de tres poblaciones del centro de Veracruz. Foresta Veracruzana, 7 (1), 31-36.

Martínez-Pérez, G., Orozco-Segovia, A. y Martorell, C. (2006). Efectividad de algunos tratamientos pre-germinativos para ocho especies leñosas de la Mixteca Alta oaxaqueña con características relevantes para la restauración. Boletín de la Sociedad Botánica de México, 79, 9-20.

Pons, T. L. (2000). Seed responses to light. En M. D. Fenner (Ed.) Seeds. The ecology of regeneration in plant communities (2nd ed.) (pp. 237-260). United Kingdom: Cabi Pub.

Rzedowski, J. (1978). Vegetación de México. México: Limusa.

Tenorio-Galindo, G., Rodríguez-Trejo, D. A. y López-Ríos, G. (2008). Efecto del tamaño y color de la semilla en la germinación de Cecropia obtusifolia Bertol (Cecropiaceae). Agrociencia, 42 (5), 585-593.

Tripathi, R. S. y Khan, M. L. (1990). Effects of seed weight and microsite characteristics on germination and seedling fitness in two species of Quercus in a subtropical west hill forest. Oikos, 57, 289-296.

Vázquez V., M. L. (1992). El género Quercus (Fagaceae) en el estado de Puebla, México. Tesis de licenciatura no publicada. Escuela Nacional de Estudios Profesionales Zaragoza, UNAM. México, D. F. 
Zavala Ch., F. y García M., E. (1996). Frutos y semillas de encinos. Chapingo, Edo. de Méx.: Universidad Autónoma Chapingo.

Zavala Ch., F. (2001). Introducción a la ecología de la regeneración natural de encinos. Chapingo, Edo. de Méx.: Universidad Autónoma Chapingo.

Zavala Ch., F. (2003). Identificación de encinos de México. Chapingo, Edo. de Méx.: Universidad Autónoma Chapingo.

Zavala Ch., F. (2004). Desecación de bellotas y su relación con la viabilidad y germinación en nueve especies de encinos mexicanos. Ciencia ergo sum, 11 (2), 177-185.
Zavala Ch., F. (2007). Guía de los encinos de la Sierra de Tepotzotlán, México. Chapingo, Edo. de Méx.: Universidad Autónoma Chapingo.

Manuscrito recibido el 12 de febrero de 2016.

Aceptado el 4 de julio de 2016.

Este documento se debe citar como:

Rodríguez-Trejo, D. A. y Pompa-García, M. (2016). Tamaño, color de nuez y sombra afectan la germinación de Quercus deserticola. Madera y Bosques, 22 (2), 67-75. 